Document downloaded from:

http://hdl.handle.net/10251/47589

This paper must be cited as:

Benajes Calvo, JV.; Bermúdez, V.; Climent, H.; Rivas Perea, ME. (2013). Instantaneous pressure measurement in pulsating high temperature internal flow in ducts. Applied Thermal Engineering. 61(2):48-54. doi:10.1016/j.applthermaleng.2013.07.033.

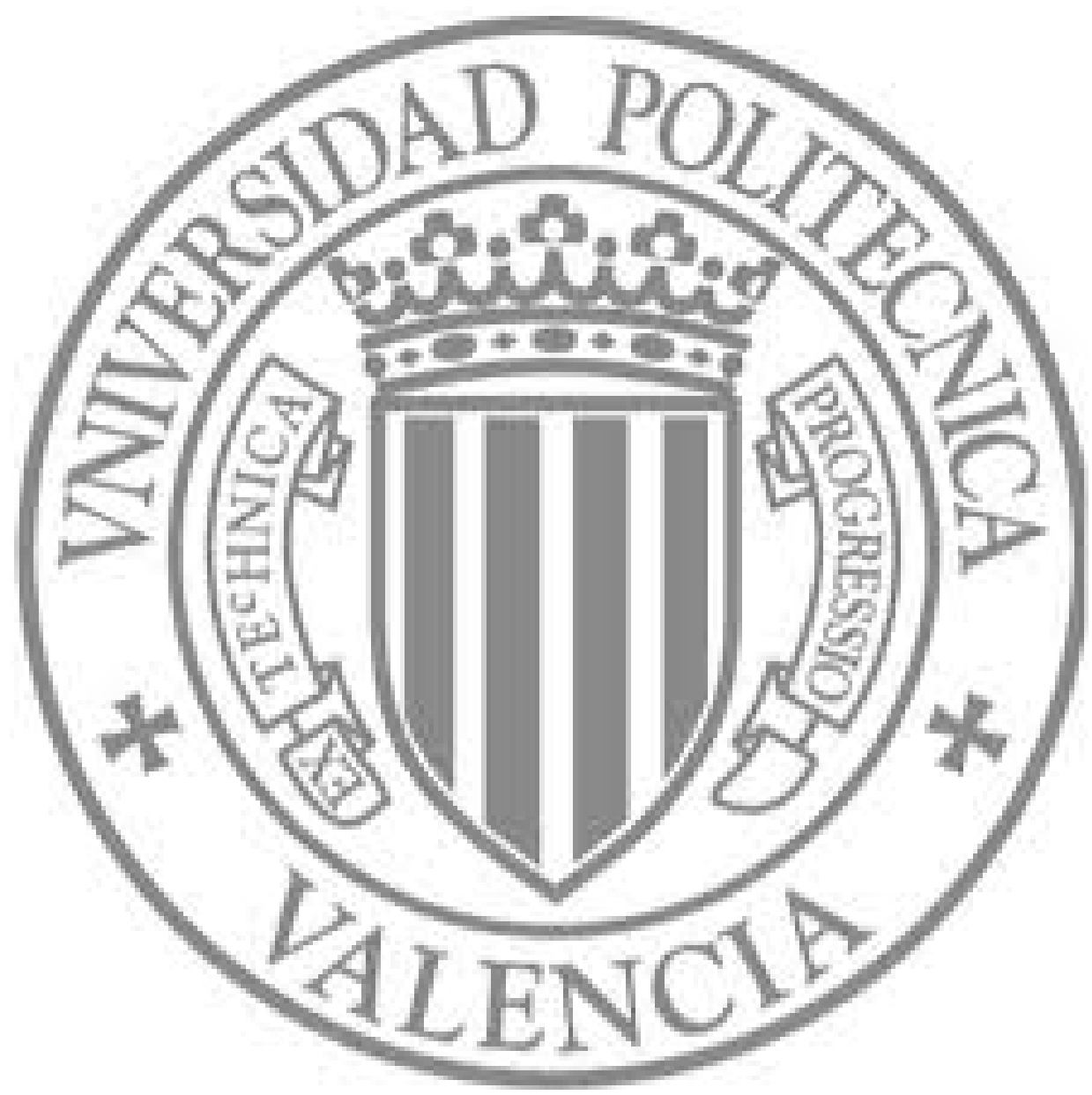

The final publication is available at

http://dx.doi.org/10.1016/j.applthermaleng.2013.07.033

Copyright Elsevier 


\title{
Instantaneous pressure measurement in pulsating high temperature internal flow in ducts
}

\author{
Jesús Benajes, Vicente Bermúdez, Héctor Climent *, Manuel Eduardo Rivas-Perea \\ CMT - Motores Térmicos. Universitat Politècnica de València.
}

* Corresponding author: hcliment@mot.upv.es Postal address: CMT Motores Térmicos, Universitat Politècnica de València. Camino de Vera s/n. 46022. Valencia. Spain.

\begin{abstract}
Research tasks in internal combustion engines often require instantaneous pressure measurement of the gases inside the exhaust manifold including absolute value information. In such environment, flow operation includes high temperature and extremely pulsating conditions. The work described in the paper identifies the problems that arise when acquiring absolute pressure with a piezoresistive transducer. A methodology based on using an additional cooler for a piezoresistive sensor placed between the duct and the original sensor cooler adaptor is proposed for dynamic pressure measurement. Specific tests have been performed in cold and hot pulsating flow in order to check the influence of the additional cooler geometry. It is possible to remove the effect of the additional cooler but the procedure depends on the the pressure in the duct. If relevant frequencies in the signal are similar to the natural frequency of the cooler, a simple filtering technique is not recommended. In this case, direct application of the acoustic theory inside ducts allows the reconstruction of the instantaneous pressure in the duct. Comparisons between the reconstructed pressure and measurements carried out with piezoelectric transducers, which do not capture the absolute value of the signal but can be placed in the duct without using an additional cooler, in hot and pulsating flow are presented. Results confirm that the method for pressure reconstruction from additional cooled piezoresistive transducers provides accurate information for instantaneous pressure measurement in hot and pulsating flow conditions inside ducts.
\end{abstract}

\section{Keywords}

Instantaneous pressure measurement; Hot pulsating flow; Piezoresistive sensor

\section{Introduction}

Instantaneous pressure measurement is an interesting tool often used in research areas in internal combustion engines. Pressure pulses inside intake and exhaust manifolds influence on engine performance in several ways. In a four-stroke engine, volumetric efficiency can be improved by tuning the intake, and pumping losses reduction is achieved with proper exhaust manifold design [1]. In two-stroke engines, the exhaust system geometry is tuned in order to configure 
the instantaneous pressure evolution in the exhaust port for improving cylinder scavenging and avoid short-circuit phenomenon [2]. Therefore, cycle-resolved pressure acquisition in intake and exhaust manifolds gives relevant information concerning internal fluid-dynamic processes in engines. This experimental information can also be helpful to develop or validate models when performing engine simulations [3].

To measure pressure an electrical signal must be generated in response to a pressure input. Typically, pressure is measured by allowing the pressure to deflect or strain a member of a transducer. This strain can then be measured in a variety of ways, using capacitive, piezoresistive and piezoelectric effects, as well as other techniques to measure displacement. In intake and exhaust systems in engine applications, either piezoresistive or piezoelectric transducers are widely employed. Piezoelectric measuring technology is used for carrying out measurement tasks with extreme requirements in terms of geometry, temperature range and dynamics. On the other hand, piezoresistive sensors are used for truly static pressure measurement. Their dynamic characteristics are also excellent but cannot withstand as high temperatures as piezoelectric devices.

Focussing on instantaneous pressure measurement in exhaust systems, flow temperature is an important issue. When the engine exhaust valves open, hot gases leave the cylinder at extremely high temperature, with averaged values up to $1000^{\circ} \mathrm{C}$, depending on the application. Piezoelectric sensors can be placed directly in the duct with the main inconvenient that absolute pressure level is not captured, which often provides important information. On the other hand piezoresistive sensors are able to register not only pressure dynamics but also the absolute value. However, they cannot be placed directly in the duct due to temperature constraints [4]. For this reason, piezoresistive sensors in hot environment applications are provided with a cooled adapter in order to reduce the temperature of the gases that are in contact with the sensor diaphragm. The performance of this cooler is valid for certain applications but, under exhaust gas flow extreme temperatures, measurement accuracy gets compromised. The design and water flow specifications of coolers adaptators that can be found on the market are very similar, so they all have accuracy problems when the sensor is placed very closed to the exhaust port. In order to solve this problem, the paper presents a methodology related to the use of an additional cooler that can be employed for acquiring absolute pressure measurements in high temperature, pulsating flow conditions, with accuracy and repeatability. Mounting techniques and geometry studies have already been carried out for in-cylinder pressure measurement using piezoresistive [5] and piezoelectric sensors [6, 7] as it is very important to develop data treatment [8] and detect measurement errors [9] in order to perform accurate combustion analysis [10]. In the paper a Kistler 4045 A2 piezoresistive sensor with the original cooler adapter 7511 provided by the manufacturer, whose main characteristics are shown in the left side of table 1, is used in order to obtain the instantaneous and absolute value of the pressure. In addition, a Kistler 6031 piezoelectric sensor with the original cooler 
adapter 6509 provided by the manufacturer, whose main characteristics are shown in the right side of the table 1 , is used to obtain the instantaneous pressure signal. A methodology based on using an additional cooler placed between the duct and the piezoresistive sensor is proposed for dynamic pressure measurement accuracy and repeatability.

The paper is structured as follows. Section 2 is devoted to the identification of the problem when using piezoresistive devices under steady and hot flow conditions. Section 3 contains the proposed solution in order to achieve accurate measurements using an additional cooler. Reconstruction of the pressure in the duct from measurements in the cooled piezoresistive sensor with the application of the acoustic theory in compressible flow in ducts is described in Section 4. Results and discussion are given in Section 5. Finally, main conclusions of the present study are presented in Section 6.

\section{Identification of the problem}

A thermal characterisation of the transducers in an air furnace up to $100^{\circ} \mathrm{C}$ reading ambient pressure was performed. Each transducer was placed with its own cooler adapter, one with manufacturer's recommended cooling flow through it and the other one with higher flow. As figure 1 depicts, significant thermal zero shift was detected during the transient cooling of the transducers from the furnace to room temperature. Thermal zero shift was greater in the case where high cooling flow was used. Thermal zero shift is the difference between the measured pressure by the sensor and the real pressure.

In summary, zero shift issue during transient cooling in the air furnace when measuring static pressure, lead to the conclusion that the root cause of the problem could be the temperature gradient at the transducer membrane. The central area of the diaphragm is directly in contact with hot gases, and the peripheral area is strongly cooled by the cooling adapter, creating a significant local temperature gradient in the membrane that affects the normal operating conditions of the transducer. When reducing the gas temperature, and thus the gradient, a smaller reading shift was detected. This problem is going to be amplified in pulsating hot flow conditions (exhaust pressure measurement) leading to false pressure measurements, so an additional cooler design is needed to solve the transducer membrane gradient problem in order to ensure measurement accuracy.

\section{Additional cooler design}

Assuming the hypothesis that temperature gradient in the diaphragm causes measurement issues in piezoresistive sensors, the objective is to try to reduce exhaust gas temperature that will be in contact with the transducer membrane. The only valid approach is to place a second cooler between the exhaust pipe and the cooling adapter 7511 provided by the manufacturer. In this way, a small and cooled volume of gas is created and direct contact of the circulating hot gases 
with the membrane is prevented. A basic rule when measuring dynamic pressure in a duct is to place the sensor surface of the transducer in the same plane as the duct wall. When there is a volume between the duct and the sensing surface, the geometry of the system changes with the addition of a volume and, therefore, the pressure wave dynamics is affected.

The instantaneous pressure in a duct is the composition of the waves travelling forward and backward in the duct. A lateral volume will introduce a boundary condition in the system with its own natural frequency. The size of the volume determines the importance of this effect: the influence is higher when the volume increases. Since the pressure is measured in the bottom section of the volume, the measured pressure will be the composition of the pressure in the duct and the pressure waves contributed by the cooler geometry. As the length of the volume shortens, the resonance frequency increases and, therefore, induces less interference with the main pressure signal at low frequency content.

Due to the pulsating characteristics of the flow in the duct, there is a fluctuating gas flow within the volume, producing an entrance of gas into the volume when the pressure gradients are positive and leaving the volume when they are negative. This flow in the volume creates a local pressure loss due to friction that distorts the instantaneous pressure in the membrane of the transducer from the pressure in the duct. To minimize this effect, the instantaneous flow entering and leaving the additional cooler should be small, leading to the conclusion that the cooler volume must be reduced to a minimum value.

In opposition to what has been stated in the last two paragraphs, taking into account the cooling performance of the additional cooler, its volume should be large to be able to reduce the exhaust gas temperature in the central part before arriving to the membrane. The combination of the effects commented before are difficult to predict or model and the design of the additional cooler turns into a trial-and-error approach keeping in mind that volume should be small but large enough for attaining cooling characteristics. For this reason the cooler is restricted to a large aspect ratio geometry. Figure 2 shows a schematic representation of the additional cooler geometry. A short and thin duct connects the duct with a small volume where the piezoresistive device is placed. Water flows outside this duct in order to reduce the temperature of the gases inside the cooling device.

\section{Pressure reconstruction}

The procedure for determining the instantaneous pressure in the duct using the measured signal from the cooled piezoresistive sensor is detailed in this section. The main idea is to translate the pressure that is measured at the closed end of the additional cooler, as seen in figure 2, to the open end, that is, the junction to the duct. The additional cooler geometry resembles a Helmholtz resonator, whose natural frequency can be obtained with [11]: 


$$
f_{n}=\frac{c}{2 \pi} \sqrt{\frac{S}{V L^{\prime}}}
$$

where $c$ is the sound velocity, $V$ is the volume of the resonator chamber, and $S$ is the neck cross section and $L$ ' is the neck length. The natural frequency of a straight duct with a single closed end is:

$$
f_{d}=\frac{c}{4 L}
$$

Therefore, a straight duct with a single closed end and the same natural frequency as the additional cooler would have a length that can be calculated with:

$$
L_{e q}=\frac{\pi}{2} \sqrt{\frac{V L^{\prime}}{S}}
$$

Figure 3 shows the transfer functions of a Helmholtz resonator, whose $V, S$ and $L$ ' values are obtained from the additional cooler geometry in figure 2, and the equivalent duct with the same natural frequency. The behaviour of both systems is very similar, at least in the frequency range that is relevant for engine operation that corresponds to frequencies lower than $1200 \mathrm{~Hz}$.

Assuming the previous analogy between the additional cooler and a straight duct (with a modified length calculated by Equation 3), the main objective is to translate the pressure measured in the closed end to the open end. This operation can be easily performed by using the acoustic theory when solving the compressible flow equations inside the equivalent duct. In fact, using the well known transfer matrix representation [12], it is possible to translate pressure and velocity from one side of a duct to the other with:

$$
\left[\begin{array}{c}
p_{1} \\
v_{1}
\end{array}\right]=\left[\begin{array}{cc}
\cos \left(k_{0} L\right) & i Y_{0} \sin \left(k_{0} L\right) \\
\left(i / Y_{0}\right) \sin \left(k_{0} L\right) & \cos \left(k_{0} L\right)
\end{array}\right]\left[\begin{array}{c}
p_{2} \\
v_{2}
\end{array}\right]
$$

where $k_{0}$ is the wave number, $L$ is the length of the duct and $Y_{0}$ the characteristic impedance.

Since pressure at the closed end is measured and velocity equals to zero, one could obtain these variables at the other end of the duct. However, $k_{0}$ and $Y_{0}$ depend on the sound velocity, which depends as well on gas temperature, which is not constant along the cooler. This issue is solved if a temperature gradient is assumed inside the cooler [13]. For temperature field determination a linear variation between exhaust gas temperature in the duct, which should be measured, and the temperature of the piezoresistive diaphragm is assumed. Following sections will detail how this value is obtained.

\section{Results and discussion}




\subsection{Thermal efficiency}

In order to evaluate the cooling capability of the additional cooler, it was tested in an engine exhaust pipe. The cooling adapter recommended by the piezoresistive manufacturer was also attached but the transducer was replaced by a dummy device with a thermocouple bounded in the centre of a thin plate placed in the same position of the transducer diaphragm. Figure 4 shows the results of these tests.

The two lines show the thermocouple readings as a function of the exhaust gas temperature with and without the additional cooler. The cooling water temperature at the outlet of the coolers was $20^{\circ} \mathrm{C}$ and the water flows were: 0.5 1/min in the piezoresistive cooling adapter and $21 / \mathrm{min}$ in the additional cooler. It can be observed that the additional cooler is able to keep a constant temperature in the core of the membrane, thus, reducing greatly the thermal gradient between the coolant and the centre of the plate. It is also interesting to note how the temperature in the membrane increases with the exhaust gas temperature. This increase is unacceptable when the additional cooler is not used and validates the hypothesis of temperature gradient causing thermal shift when operating at pulsating hot flow conditions.

\subsection{Pulsating and cold flow conditions}

Specific tests were designed in the pulsating test flow rig described in figure 5 for evaluating the influence of the additional cooler on the instantaneous pressure existing in the pipe. Two piezoresistive transducers were placed one opposite the other in a cross section of the straight pipe. Two tests were performed: (A) the two transducers are placed without using the additional cooler, and (B) one transducer is placed without the additional cooler while the other one is attached to it. Since these tests are carried out under cold flow conditions, measurements from the transducers without the additional cooler are reliable.

Results at $900 \mathrm{rpm}$ are presented in figure 6 . The influence of the additional cooler on the pressure in the duct can be observed by comparing the measurements obtained from transducers without using the cooler in tests $\mathrm{A}$ and $\mathrm{B}$. The influence of the volume on the main signal is not significant as it is observed in the upper plot in figure 6 . It is important to highlight that the two signals compared in these figures correspond to two different tests (A and B) and, therefore, the repeatability error of the test rig is included.

The differences on the measured pressure signals (in the duct and in the volume) are detected when plotting the results of test B. As the bottom graph plots, the effect of the additional cooler is clearly observed. Small high-frequency peaks appear on the instantaneous pressure registered in the piezoresistive device placed in the additional cooler. The frequency of theses peaks correspond to the natural frequency of the additional cooler, which is about $600 \mathrm{~Hz}$ using the geometry of the cooler, the sound velocity at room temperature and Equation 1. Figure 7 shows the spectra of the 
measurements in test $\mathrm{B}$, where differences in the range of $600 \mathrm{~Hz}$ appear in the signals with and without additional cooler. An important conclusion from these tests is that the ripple induced by the volume is not translated into the main signal.

\subsection{Pulsating and hot flow conditions}

In this section, pressure measurements in a straight duct in the exhaust system of two different engines are presented. Both are gasoline engines, which attain higher exhaust temperature values than Diesel units. One is a four-stroke automotive engine while the other one is a small two-stroke engine. Both provide an exhaust pulse with an important amplitude peak at the beginning of the exhaust period. Two-stroke small engine testing is interesting since frequency content in the exhaust pressure is high due to: (a) one combustion process every engine revolution, and (b) engine speed achieves higher values than in an automotive $4 \mathrm{~S}$ engine.

Instantaneous pressure measurements are performed as follows: a piezoresistive sensor with the additional cooler and a piezoelectric sensor, whose main characteristics are shown in the right of table 1 , were placed at the same cross section in the duct measurement point as depicted in figure 8. A piezoelectric device measures instantaneous pressure in the duct without needing the additional cooler since it is able to withstand extreme exhaust gas temperatures. The piezoelectric signal average value is corrected taking into account the piezoresistive measurement.

Figure 9 shows pressure traces in the crank-angle domain measured in the exhaust duct of the $4 \mathrm{~S}$ automotive engine. Top plots present the results of the piezoresistive and piezoelectric sensors at full load and two engine speeds, 3500 and $5000 \mathrm{rpm}$. As already detected in the previous section, a small ripple arises in the piezoresistive signal due to the additional cooler geometry. Applying the transfer matrix methodology to this signal, the calculated pressure at the open end of the cooler, i.e. in the duct, is obtained. Bottom graphs depict the results from the pressure reconstruction in the duct, which are compared with the piezoelectric measurements. There is a good correlation between calculated and measured data in the duct. Discrepancies between piezoresistive and piezoelectric measurements increase with engine speed since the frequency content of the exhaust pressure approaches to the natural frequency of the cooler. Even at high engine speeds, application of the methodology provides good accuracy.

Instead of using the acoustic theory to reconstruct the signal, the results of the application of a simple low-pass filter post-processing to the piezoresistive measurements are shown in figure 10. Calculated and filtered results are plotted against piezoelectric measurements; an exact matching would show all the points in the $45^{\circ}$ bisecting line. Filtering technique is best suited for low engine speeds although results using the acoustic theory provide better correlations than 
filtering procedure. Moreover, choosing an appropriate cut-off frequency is not evident since it should be lower than the natural frequency of the cooler but large enough in order to avoid signal information loss.

Results for the instantaneous exhaust pressure in the $2 \mathrm{~S}$ engine are shown in a similar way in figure 11 , where the engine was running at 4500 and $6900 \mathrm{rpm}$. In this case, differences between piezoelectric and piezoresistive measurements are more evident as observed in the upper plots. The reconstruction of the piezoresistive signal provides the calculated data, which are also compared with the piezoelectric measurement. Results show good agreement in crank-angle resolution except when the pressure pulse, due to the opening of the exhaust port, is very steep. As figure 12 presents, filtering post-processing is definitely not convenient in this application due to high frequency content in the exhaust pressure signals. The natural frequency of the piezoresistive cooler is not high enough and choosing the cut-off frequency is critical. Increasing the natural frequency of the cooler is not a valid solution since it involves the reduction of its length, which decreases the thermal performance of the additional cooler.

\section{Conclusions}

An experimental methodology was followed in order to identify the source of the zero and sensitivity shifts of the piezoresistive transducers for dynamic pressure measurement when operating under engine exhaust conditions. Temperature gradient in the membrane of the pressure sensor is a key issue for accurate and repetitive measurements. Therefore, the use of an additional cooler placed between the duct and the sensor original cooler was proposed.

Specific tests demonstrated that the additional cooler geometry does not influence on the pressure inside the duct although the pressure registered by the cooled piezoresistive sensor may differ from the pressure in the duct. It is possible to remove the effect of the additional cooler on the signal that is registered with the cooled piezoresistive transducer but the procedure depends on the frequency content of the pressure in the duct.

If relevant frequencies in the duct pressure are lower than the natural frequency of the cooler, a low-pass filter can be applied in order to remove the ripple added by the cooler. However, if relevant frequencies in the signal are similar to the natural frequency of the cooler, a simple filtering technique provides inaccurate results. In this case, direct application of the linear theory for solving the behaviour of the compressible flow within a one-dimensional approach inside ducts allows the reconstruction of the instantaneous pressure in the duct by using the registered signal in the cooled piezoresistive device. Low-pass filtering technique can be applied in four stroke engines running at low engine speeds but, at high speeds or in small two stroke engines, signal reconstruction using the acoustic theory, via transfer matrix representation, provides better results. 
Results confirm that the method for pressure reconstruction from the additional cooled piezoresistive transducer, which account for absolute pressure value, provides accurate information for instantaneous pressure measurement in hot and pulsating flows inside ducts.

\section{References}

[1]. Blair G, Mackey D, Ashe M, Chatfield G, Exhaust Tuning on a Four-Stroke Engine, Experimentation and Simulation, SAE Paper (2001) 2001-12-01.

[2]. Galindo J, Serrano J R, Climent H, Tiseira A, Analysis of gas-dynamic effects and its relevance in compact exhaust system design of small two-stroke engines, International Journal of Automotive Technology 8(4): (2007) 403-11.

[3]. Stefano Cereda, Roberto Bossù, Massimiliano Gambarotto and Costanza Pazé 1-D Modeling and Room Temperature Experimental Measurements of the Exhaust System Backpressure: Limits and Advantages in the Prediction of Backpressure, SAE Paper (2008) 2008-01-0676.

[4]. Kunimatsu M, Mizuide $\mathrm{T}$ and Yamame $\mathrm{K}$, Measurement of dynamic pressure using piezoelectric sensors at extremely low temperatures, JSAE Review 22: (2001) 553-8.

[5]. H. Angström, Cylinder pressure indicating with multiple transducer, accurate TDC-evaluating, zero levels and analysis of mechanical vibrations, in: 3 Internationales indiziersymposium, Proceedings, (1998) 103-108.

[6]. Andrew L. Randolph, Cylinder-Pressure-Transducer Mounting Techniques to Maximize Data Accuracy, SAE Paper (1990) 1990-01-0644.

[7]. Richard S. Davis and Gary J. Patterson, Cylinder Pressure Data Quality Checks and Procedures to Maximise Data Accuracy, SAE paper (2006) 2006-01-1346.

[8]. F. Payri, P. Olmeda, C. Guardiola, J. Martín. Adaptative determination of cut-off frequencies for filtering the incylinder pressure in diesel engines combustion analysis. Applied Thermal Engineering, 31 (2011) 2869-2876.

[9]. F. Payri, S. Molina, J. Martín, O. Armas. Influence of measurement errors and estimated parameters on combustion diagnosis. Applied Thermal Engineering, 26 (2006) 226-236.

[10]. M. Lapuerta, O. Armas, J.J. Hernandez. Diagnosis of DI Diesel combustion from in-cylinder pressure signal by estimation of mean thermodynamic properties of the gas. Applied Thermal Engineering, 19 (1999) 516-529.

[11]. Lawrence E, Austin R, Coppens A, Fundamentals of acoustics, John Wiley \& Sons, Inc. New York, USA, 2000.

[12]. Crocker M J, Handbook of acoustics, John Wiley \& Sons, Inc. New York, USA, 1998. 
[13]. Sujith R I, Transfer matrix of a uniform duct with an axial mean temperature gradient, Journal of the Acoustical Society of America 100(4): (1996) 2540-42. 


\begin{tabular}{llll}
\hline Technical data & units & Piezoresistive & Piezoelectric \\
\hline Range & bar (abs) & $0-2$ & $0-250$ \\
Overload & bar (abs) & 5 & 350 \\
Sensitivity & & $250 \mathrm{mV} / \mathrm{bar}$ & $-14 \mathrm{pC} /$ bar \\
Natural frequency & $\mathrm{kHz}$ & $>20$ & 160 \\
Linearity & $\pm \% / \mathrm{FSO}$ & $<5$ & $=1$ \\
Thermal zero shift & $\pm \% / \mathrm{FSO}$ & $<0.5$ & $\mathrm{n} . \mathrm{a}$ \\
Thermal sensitivity shift & $\pm \%$ & $<1$ & $<2$ \\
Temperature range & ${ }^{\circ} \mathrm{C}$ & $20-140$ & $-196-200$
\end{tabular}

Table 1. Technical data of piezoresistive and piezoelectric pressure sensors

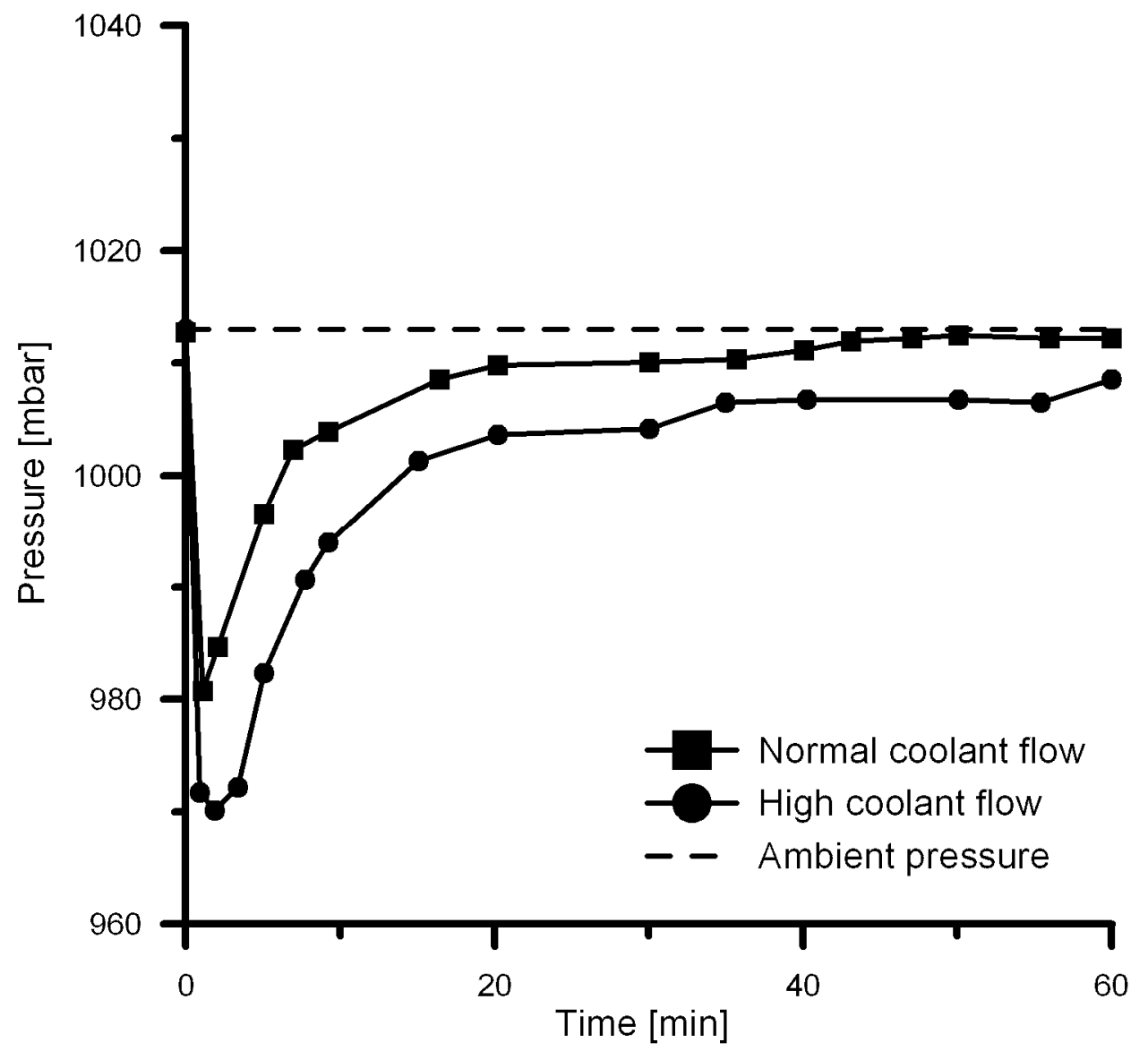

Fig 1. Ambient pressure measurement during transient cooling with high coolant flow (circles) and normal coolant flow (squares) 


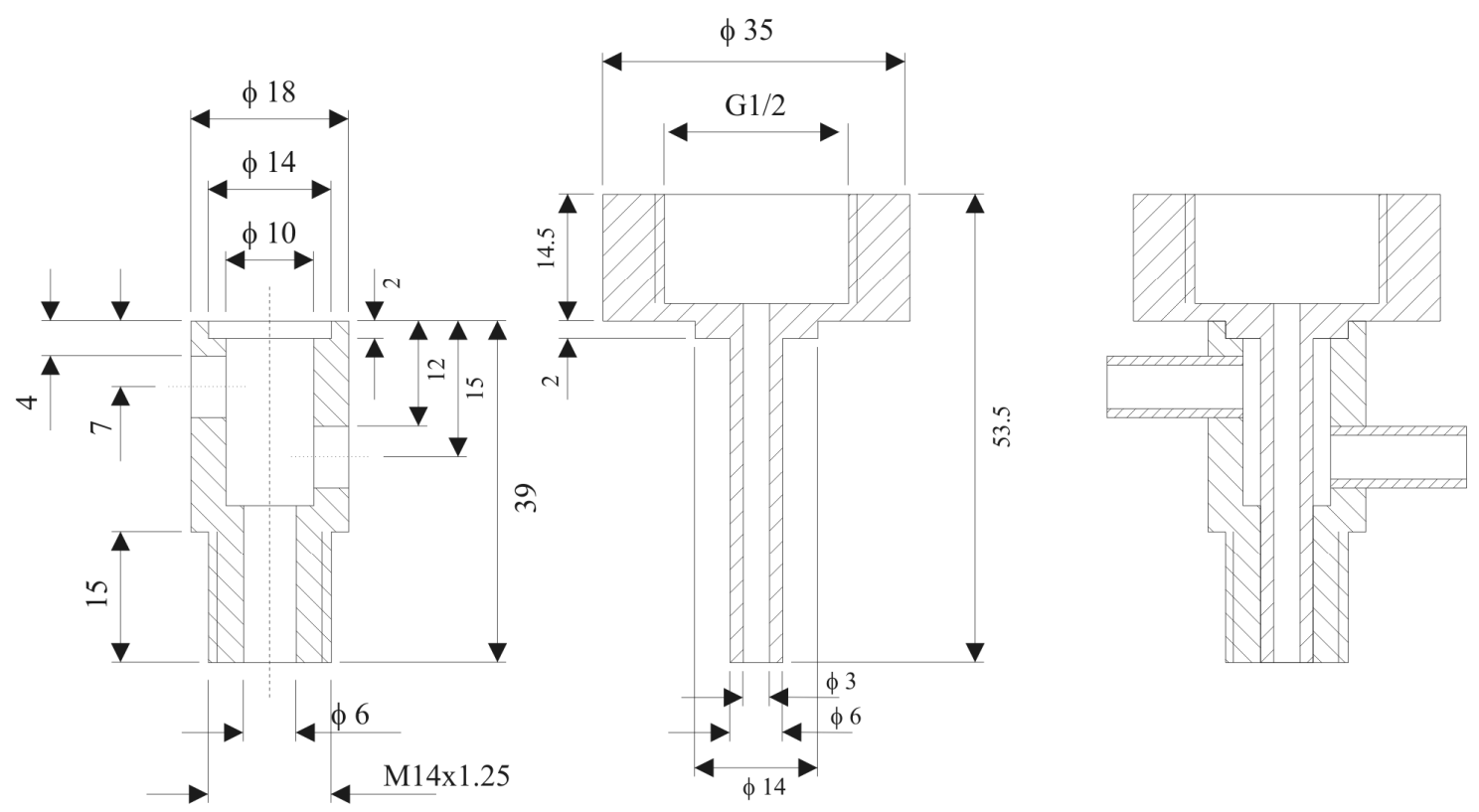

Fig 2. Schematic representation of the additional cooler geometry

\section{Frequency response [-]}

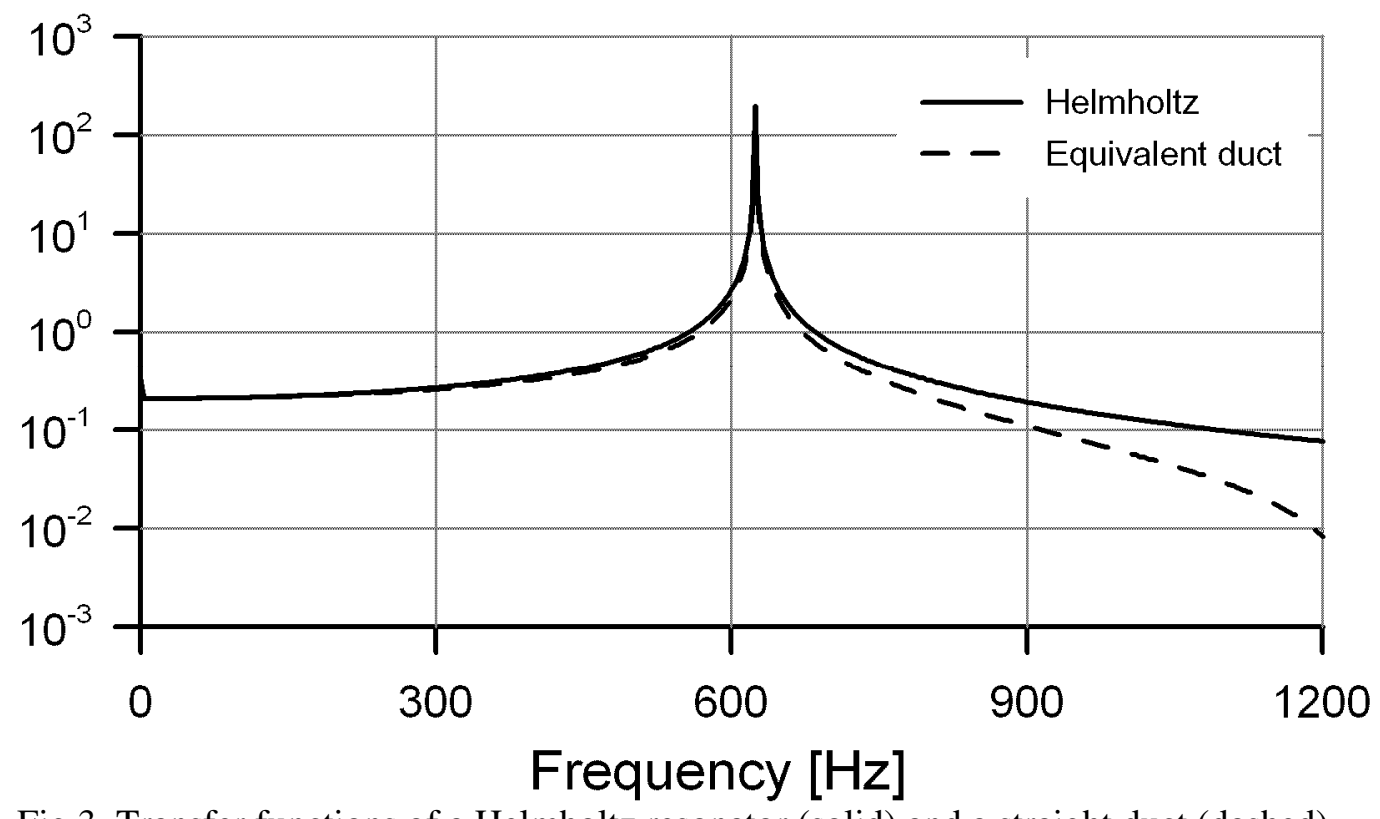

Fig 3. Transfer functions of a Helmholtz resonator (solid) and a straight duct (dashed) 


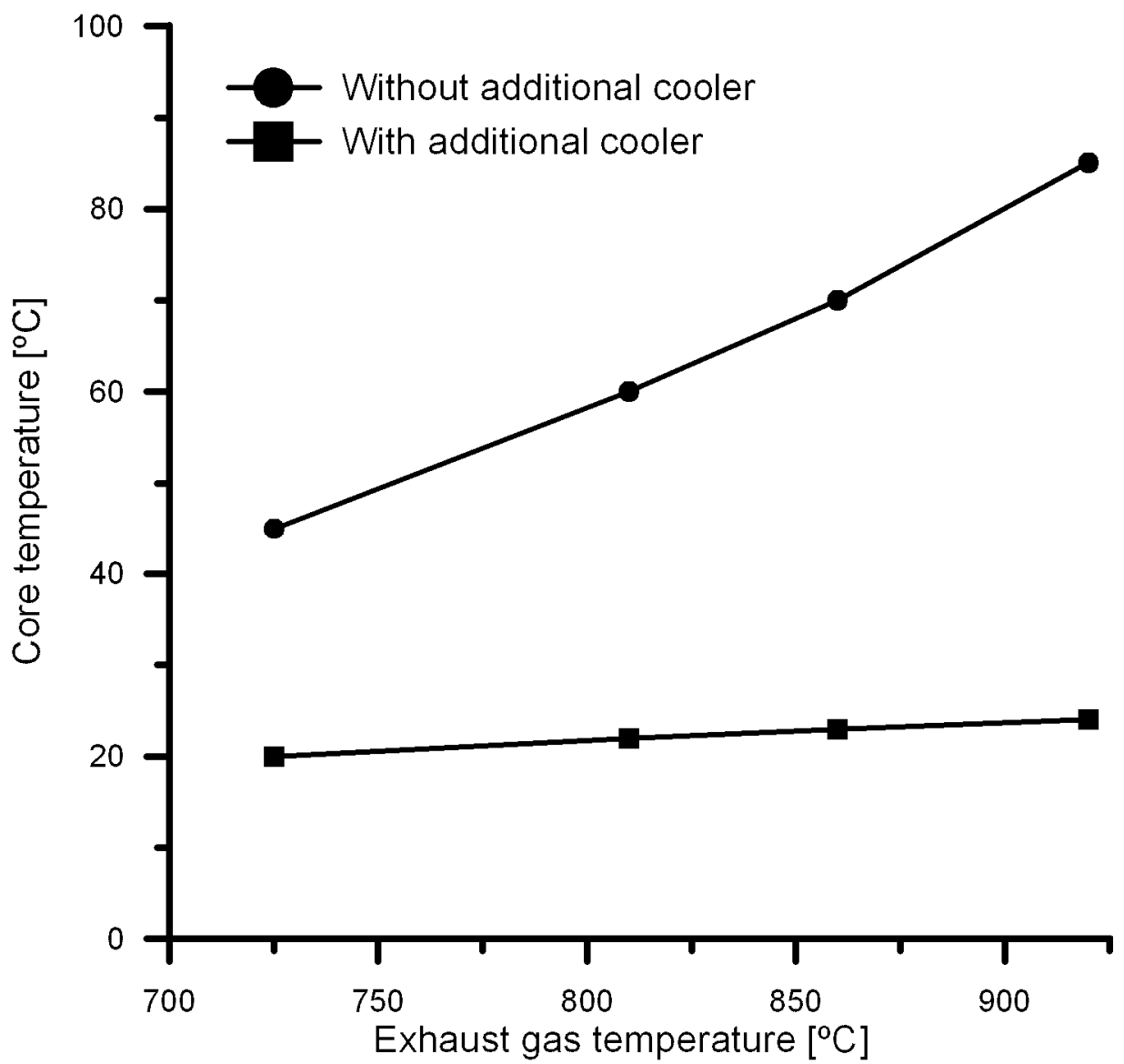

Fig 4. Membrane temperature with the additional cooler (squares) and with the original configuration (circles)

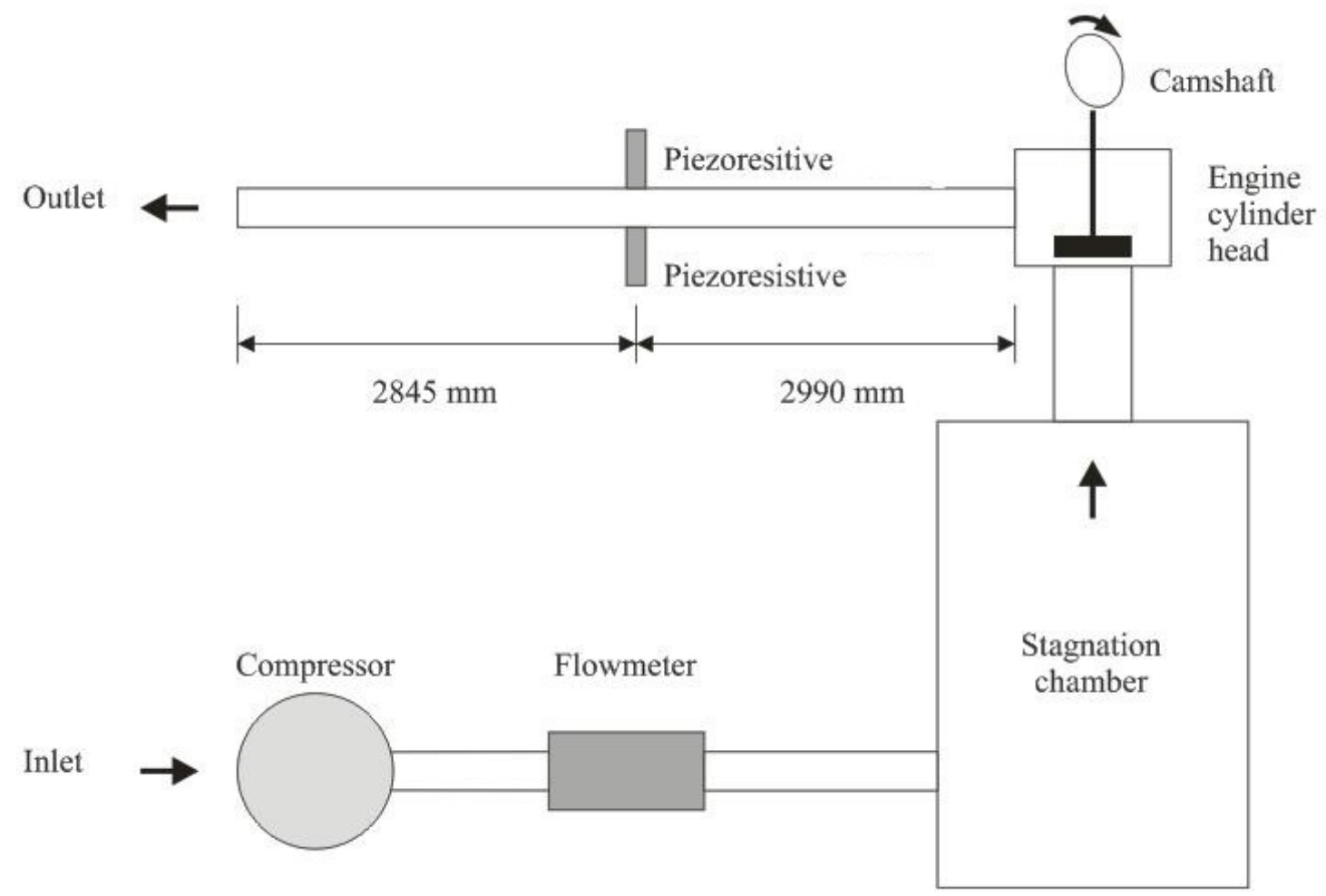

Fig 5. Cold and pulsating flow test rig schematic layout 


\section{Pressure [bar]}
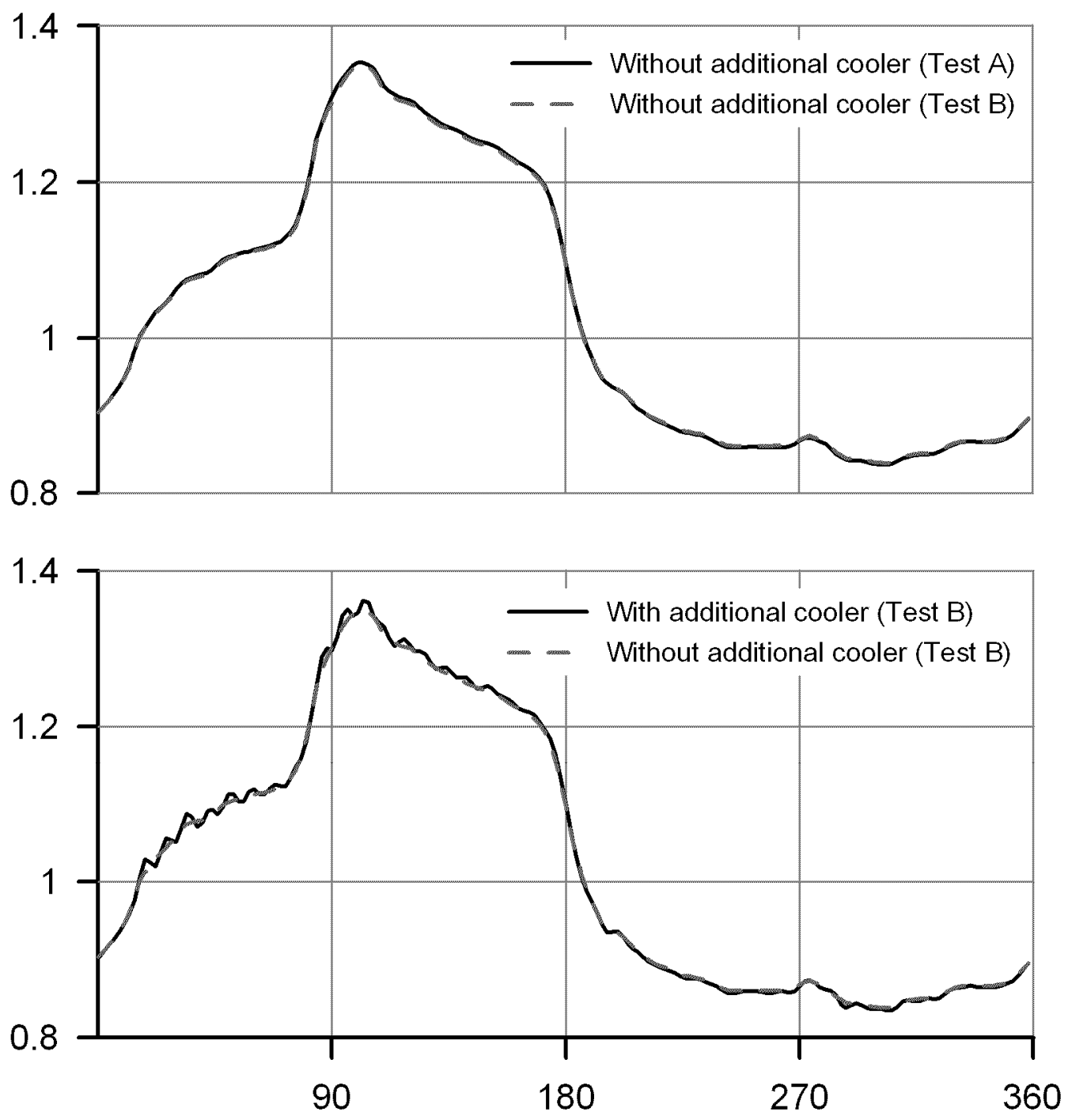

Crank Angle $\left[^{\circ}\right]$

Fig 6. Pressure measurements in pulsating and cold flow conditions at $900 \mathrm{rpm}$

\section{Amplitude [bar]}

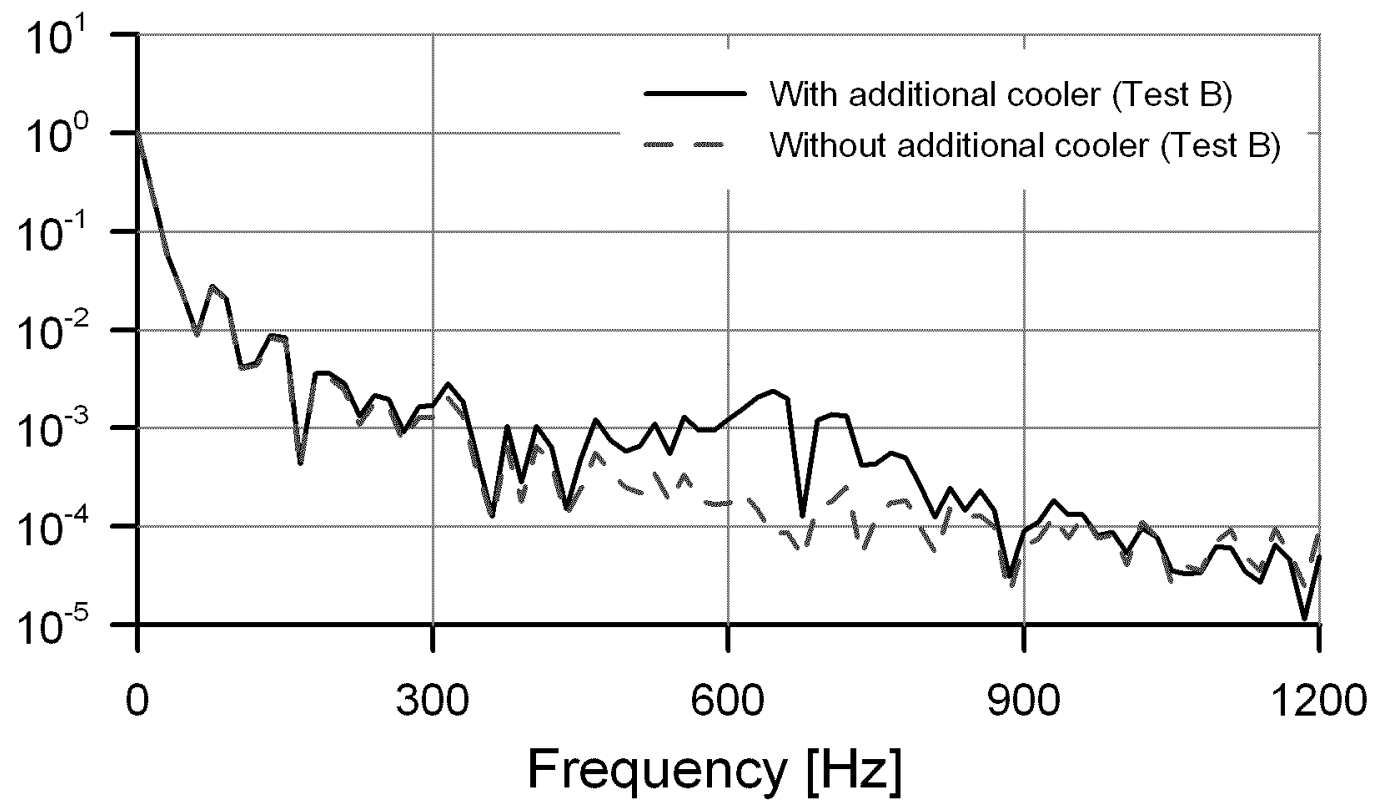

Fig 7. Spectra of measurements in cold flow conditions test B at $900 \mathrm{rpm}$ 


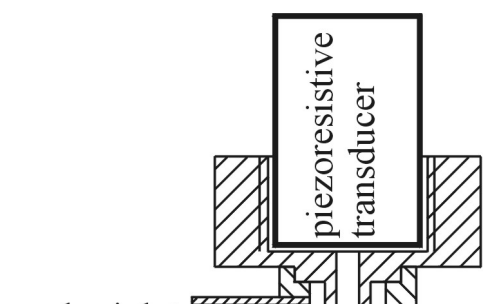

cooler inlet

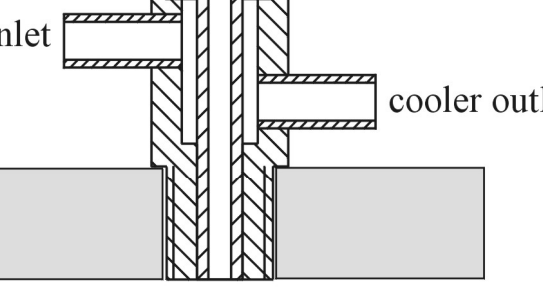

exhaust gases $\longrightarrow$

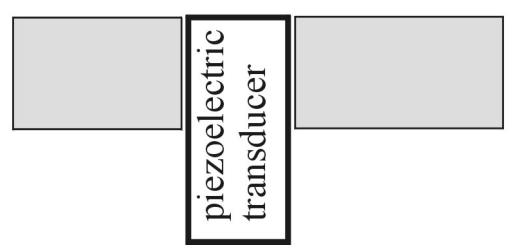

Fig 8. Arrangement of pressure measurement devices in pulsating and hot flow conditions
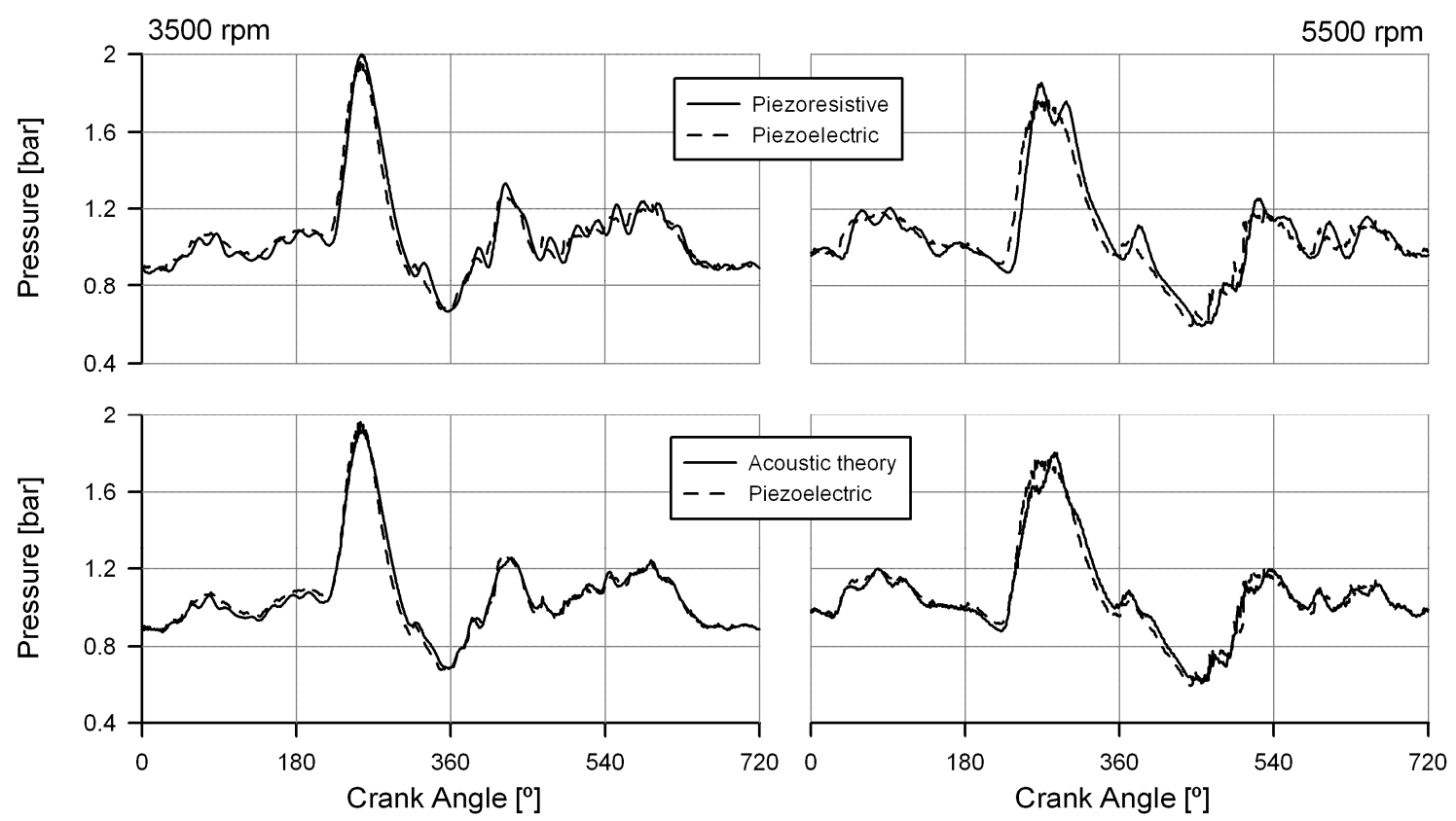

Fig 9. Pressure reconstruction and measurements in pulsating and hot flow conditions in a $4 \mathrm{~S}$ engine 


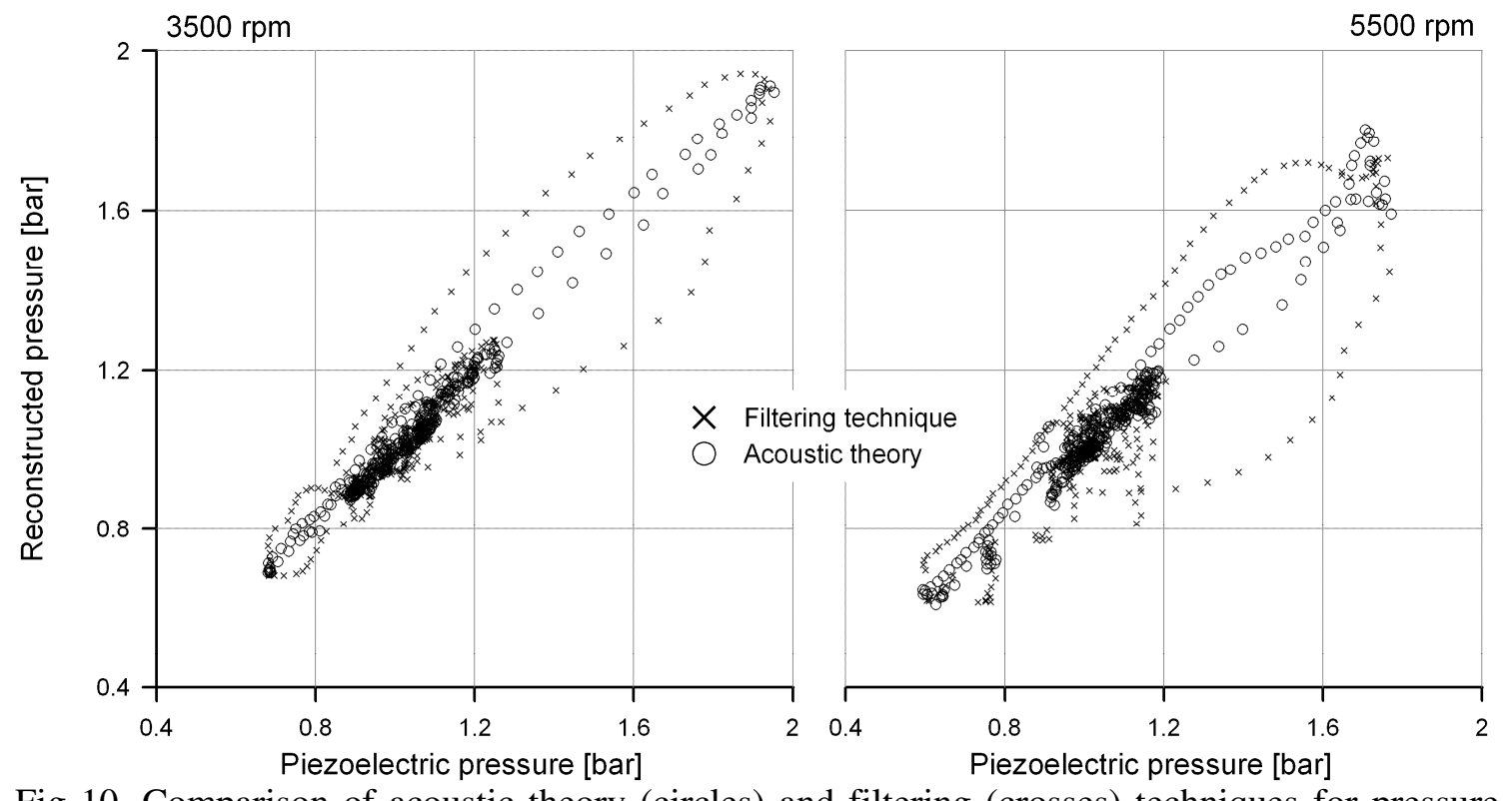

Fig 10. Comparison of acoustic theory (circles) and filtering (crosses) techniques for pressure reconstruction in a $4 \mathrm{~S}$ engine
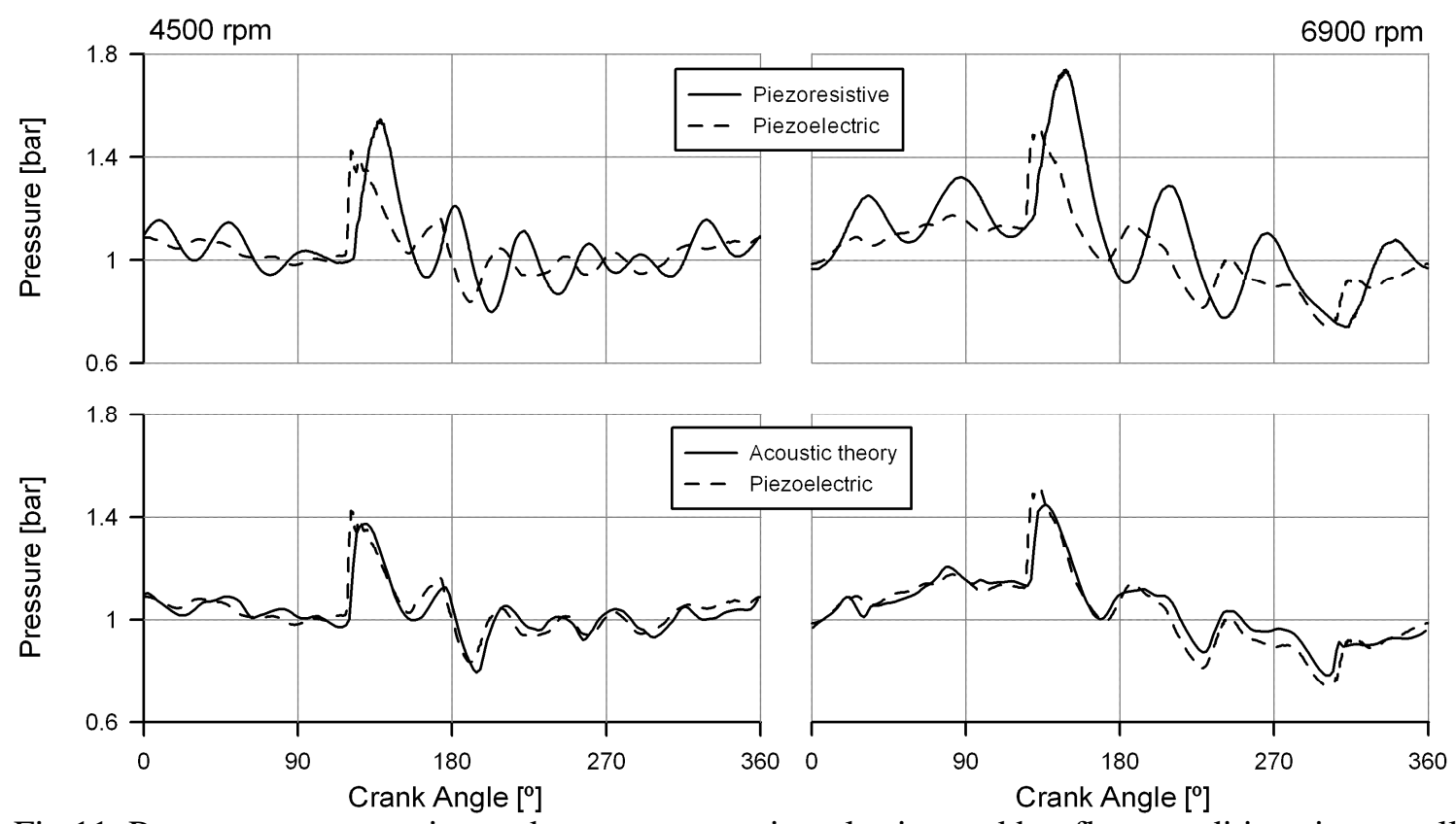

Fig 11. Pressure reconstruction and measurements in pulsating and hot flow conditions in a small $2 \mathrm{~S}$ engine 


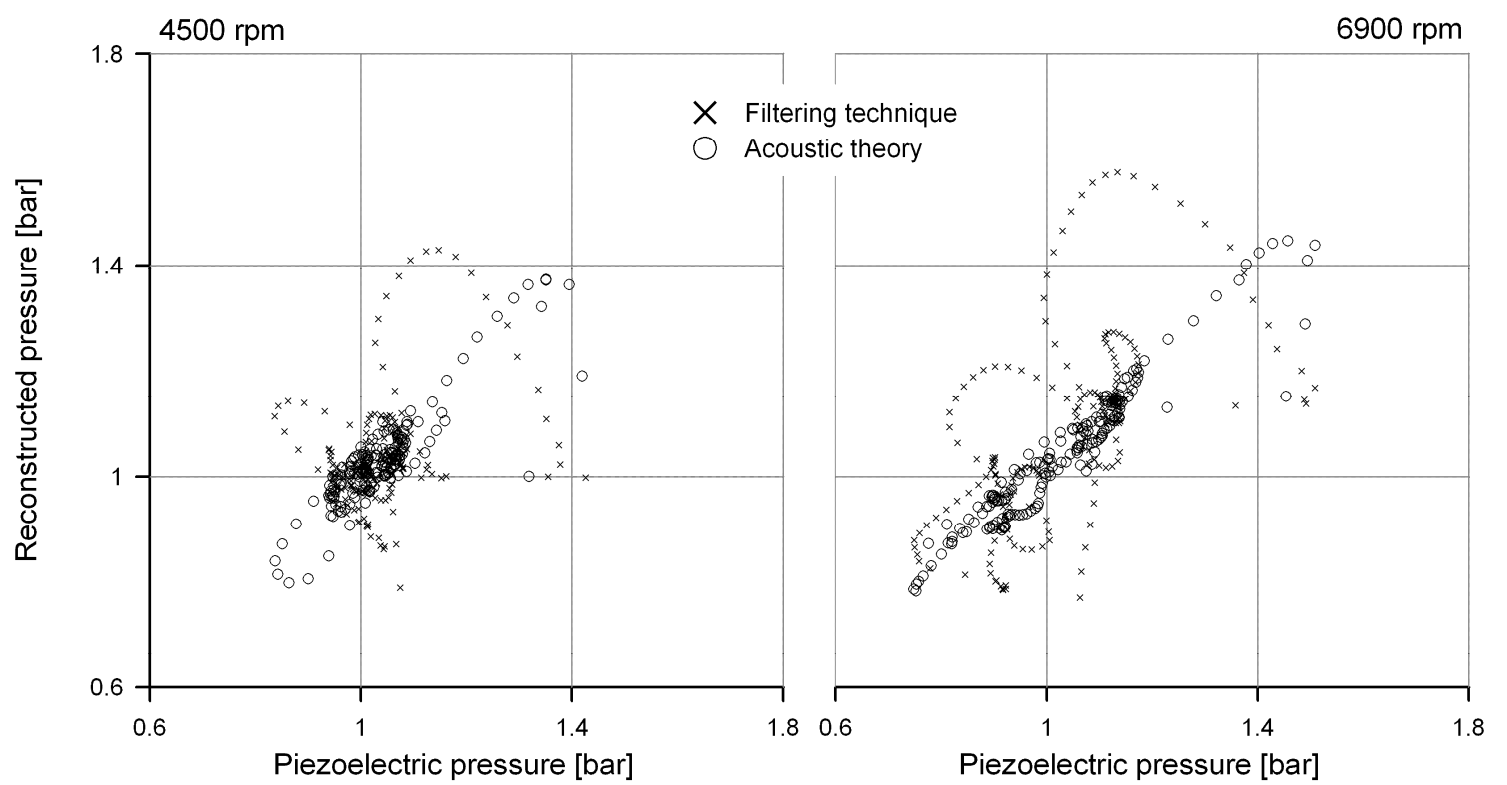

Fig 12. Comparison of acoustic theory (circles) and filtering (crosses) techniques for pressure reconstruction in a small $2 \mathrm{~S}$ engine 